\title{
Spectral-domain optical coherence tomography in healed ocular toxoplasmosis
}

\author{
Sandeep Saxena $\cdot$ Ramit A. K. Rastogi • \\ Kuldeep Vishvkarma • Sanjiv Hansraj • \\ Carsten H. Meyer
}

Received: 31 March 2011 /Accepted: 27 July 2011 / Published online: 10 August 2011

(C) Springer Science+Business Media, LLC 2011

\section{Introduction}

Toxoplasmosis is the most common cause of infectious retinitis in immunocompetent individuals. The seroprevalence of Toxoplasma gondii is different throughout the world. Among this positive population, only $1 \%$ has meaningful chorioretinitis scars. Ocular toxoplasmosis is sometimes a benign and self-limiting disease. It can also cause central or total visual loss. Legal blindness occurs in nearly one quarter of the affected eyes. It is caused either directly by involvement of the macula or optic nerve or indirectly by complications secondary to inflammation $[1,2]$.

Spectral-domain optical coherence tomography (SD-OCT) in active toxoplasmosis enabled identification of morphological features underestimated on clinical examination in patients with ocular toxoplasmosis. Spectral-domain optical coherence tomography has revealed diffuse macular edema, vitreomacular traction, maculoschisis, focal choriocapillaris/ choroidal relative hyper-reflectivity, and posterior vitreous detachment [3].

Typical congenital toxoplasmic retinochoroiditis presents as a macular cicatricial lesion. SD-OCT in two cases of healed toxoplasmosis is reported for the first time.

S. Saxena $(\bowtie) \cdot$ R. A. K. Rastogi $\cdot$ K. Vishvkarma Department of Ophthalmology, CSM Medical University (Erstwhile King George's Medical University),

Lucknow, Uttar Pradesh, India

e-mail: sandeepsaxena2020@yahoo.com

\section{S. Hansraj}

Mansarovar Eye Hospital,

Lucknow, India

C. H. Meyer

Department of Ophthalmology, University of Bonn,

Bonn, Germany

\section{Material and methods}

Two consecutive cases of healed toxoplasmosis were included. Elevated IgG levels for $T$. gondii were detected using enzyme-linked immunosorbent assay. SD-OCT was performed using Copernicus SD-OCT (Optopol, Poland). Three-dimensional imaging was also performed using SD-OCT.

\section{Case reports}

Case 1 A 24-year-old male presented with diminution of vision in his left eye for past several years. His visual acuity was 20/20 OD and counting fingers OS. Slit lamp examination results were unremarkable for both eyes. Fundus examination of his right eye was normal, but fundus examination of his left eye revealed a large healed excavated scar at the posterior pole having well-defined borders with central retinochoroidal atrophy and peripheral retinal pigment epithelial hyperplasia (Fig. 1a). SD-OCT of the left eye was performed. At the center of the lesion, a 6-mm horizontal scan showed splitting of retina at the level of outer nuclear layer. Discontinuation of photoreceptor layer, inner-segment-outer-segment junction, and hyper-reflective retinal pigment epithelium was observed (Fig. 1b). Retinal nerve fiber layer was found to be absent. Retinal thickness of $242 \mu \mathrm{m}$ was observed prior to retinal splitting. Retinal thickness at the edges of inner retinal layer break was observed to be 106 and $131 \mu \mathrm{m}$, respectively. Choriocapillaris/choroidal/scleral relative hyper-reflectivity were also observed in the center of the lesion. On threedimensional retinal imaging, an excavated scar was evident. The retinal pigment epithelium deformation map showed increased retinal pigment epithelium thickness in the peripheral area with loss of retinal pigment epithelium in the center of the lesion (Fig. 1c). 

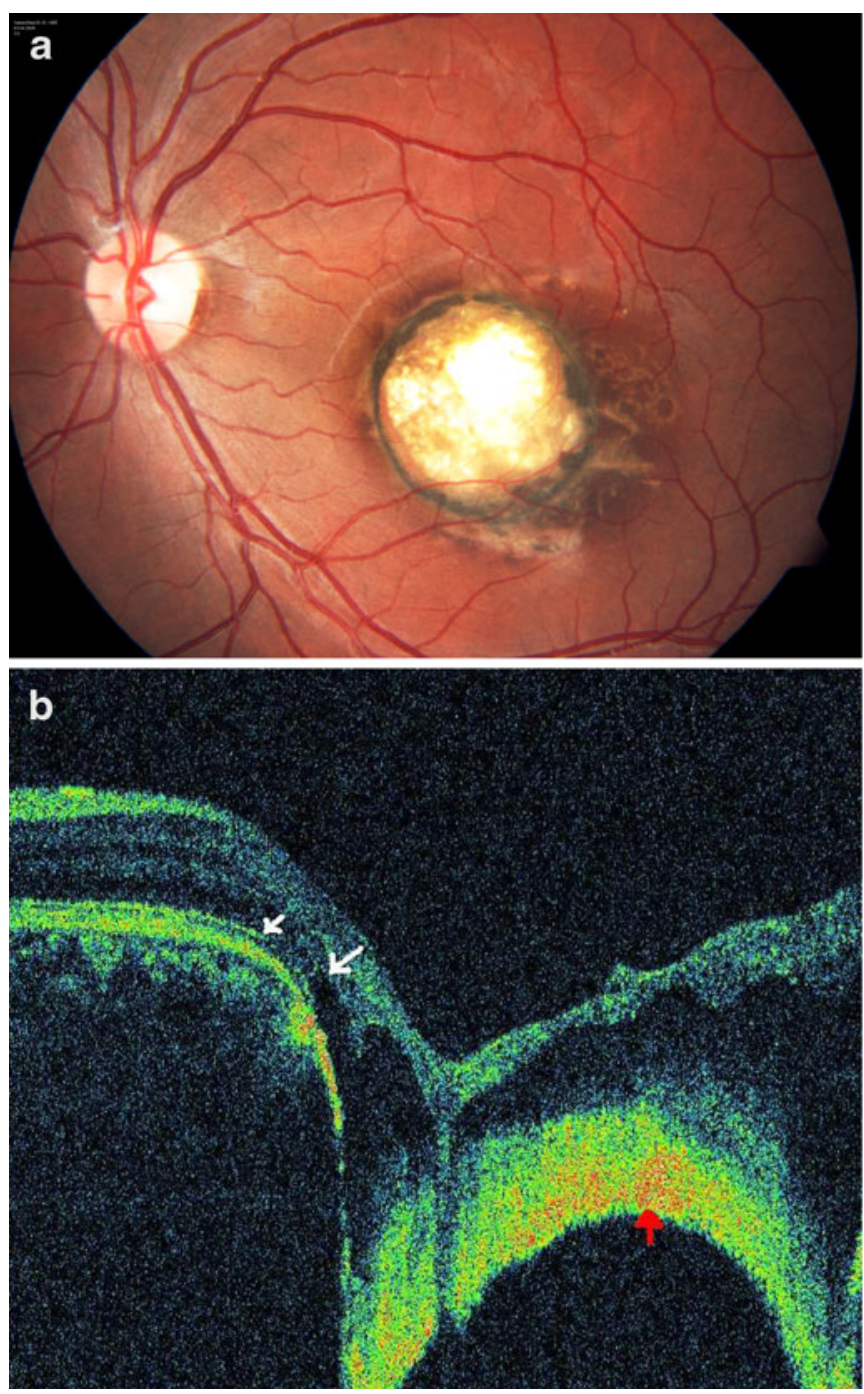

Fig. 1 a Color fundus photograph shows a large healed excavated scar at the posterior pole with central retinochoroidal atrophy and peripheral retinal pigment epithelial hyperplasia. b Spectral-domain optical coherence tomography on-line scan through the center of the lesion shows splitting of the retinal layers at the level of outer nuclear layer (large white arrow) along with the discontinuation of photoreceptor layer, inner-

Case 2 A 26-year-old male presented with diminution of vision in his right eye for past several years. His visual acuity was counting fingers OD and 20/30 OS. Slit lamp examination results were unremarkable for both eyes. Fundus examination of his left eye was normal, but fundus examination of his right eye revealed a large healed excavated scar at the posterior pole having well-defined borders with central retinochoroidal atrophy and peripheral retinal pigment epithelial hyperplasia.

SD-OCT was performed; a 6-mm horizontal scan of the right eye revealed an excavated lesion. Hyper-reflective retinal pigment epithelium was observed to be extending to the base of the lesion. Choriocapillaris/choroidal relative hyperreflectivity was also observed in the center of the lesion

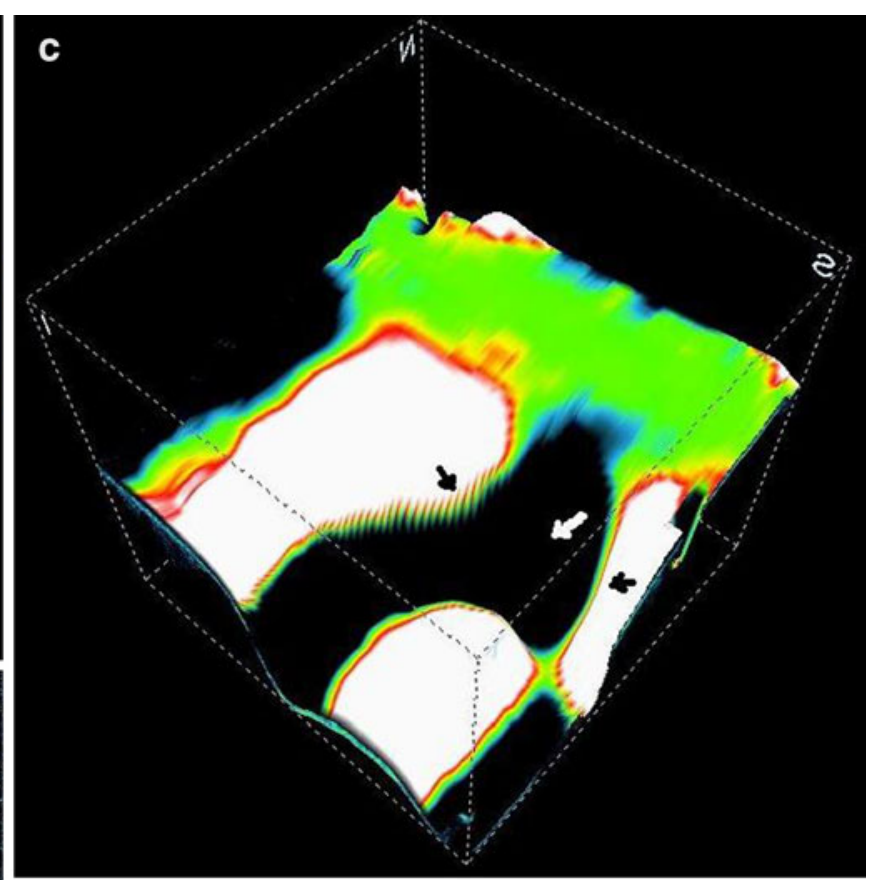

segment-outer-segment junction (small white arrow). Choriocapillaris/ choroidal/scleral relative hyper-reflectivity was also observed in the center of lesion (red arrow). c Spectral-domain optical coherence tomography retinal pigment epithelium deformation map shows increased retinal pigment epithelium thickness in the peripheral area (black arrows) with loss of retinal pigment epithelium in the center of the lesion (white arrow)

(Fig. 2a). On three-dimensional retinal imaging, a comprehensive image of an excavated scar was evident (Fig. 2b).

\section{Discussion}

Congenital toxoplasmic retinochoroiditis presents as a cicatricial lesion in the macula. Histopathologically, healed retinochoroiditis lesion of toxoplasmosis shows severe destruction in the area of infection and presence of chorioretinal adhesion [4].

Optical coherence tomography has demonstrated a variety of characteristic morphological changes, which 

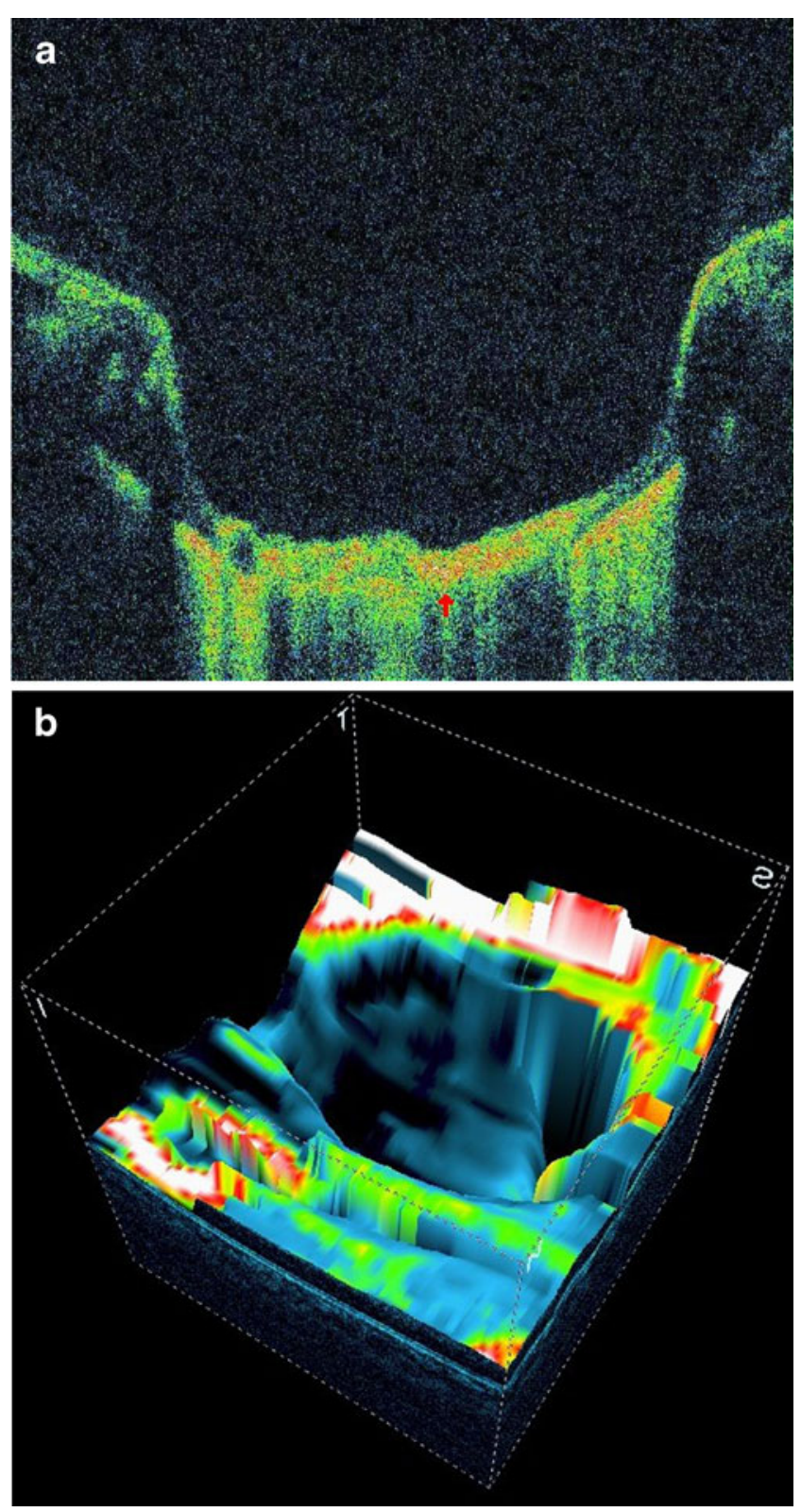

Fig. 2 a Spectral-domain optical coherence tomography horizontal $6 \mathrm{~mm}$ scan at the center of the lesion shows an excavated area with hyper-reflective retinal pigment epithelium extending to the base of the lesion and central hyper-reflectivity (red arrow). b Spectral-domain optical coherence tomography on three-dimensional retinal imaging shows a comprehensive image of an excavated scar

may point towards a specific disease process in posterior uveitis [5]. Optical coherence tomography imaging can distinguish between active and scarred toxoplasmic lesions. Optical coherence tomography provides quantitative measurements of retinal thickness, which could be useful in future prospective studies, to guide therapeutic decisions and to monitor the efficacy of treatments [6].

Imaging of congenital toxoplasmosis macular scars with optical coherence tomography reveals retinal thinning, retinal pigment epithelial hyper-reflectivity, excavation, intraretinal cysts, and fibrosis. Patients with better than expected vision have either parafoveal lesions or an intact neurosensory layer. The most characteristic OCT features include prominent retinal thinning, retinal pigment epithelium hyper-reflectivity, and excavation of varying severity. OCT imaging has been found to be helpful in explaining better than expected vision [7].

Splitting of retinal layers at the level of outer nuclear layer with discontinuation of the inner-outer-segment junction and photoreceptor layer was observed, in the first case, which was not evident clinically. Maculoschisis was also observed in healed toxoplasmosis as has been described in active toxoplasmosis [3]. Choriocapillaris/ choroidal relative hyper-reflectivity was observed in both the cases, which corresponded clinically to the area of retinochoroidal atrophy. Excavated scar was clearly evident in both the cases on three-dimensional retinal imaging as visualized on stereoscopic fundus examination. Threedimensional retinal imaging helps in documenting comprehensive excavated lesion in healed toxoplasmosis.

SD-OCT helped in elucidating morphological changes in lesions of healed toxoplasmosis for the first time, that were not apparent on clinical examination, which may expand the clinical spectrum of the disease.

\section{References}

1. Bosch-Driessen LH, Karimi S, Stilma JS, Rothova A. Retinal detachment in ocular toxoplasmosis. Ophthalmology. 2000;107:36-40.

2. Mets MB, Holfels E, Boyer KM, et al. Eye manifestations of congenital toxoplasmosis. Am J Ophthalmol. 1997;123:1-16.

3. Orefice JL, Costa RA, Orefice F, Campos W, da Costa-Lima D, Jr SIU. Vitreoretinal morphology in active ocular toxoplasmosis: a prospective study by optical coherence tomography. $\mathrm{Br} \mathrm{J}$ Ophthalmol. 2007;91:773-80.

4. Green WR. Retina: inflammatory disease and conditions. In: Spencer WH, editor. Ophthalmic pathology. An atlas and text book, vol. 2. Philadelphia: WB Saunders; 1985. p. 730-91.

5. Gallagher MJ, Yilmaz T, Cervantes-Castañeda RA, Foster CS. The characteristic features of optical coherence tomography in posterior uveitis. Br J Ophthalmol. 2007;91:1680-5.

6. Monnet D, Averous K, Delair E, Brézin AP. Optical coherence tomography in ocular toxoplasmosis. Int J Med Sci. 2009;6:137-8.

7. Garg S, Mets MB, Bearelly S, Mets R. Imaging of congenital toxoplasmosis macular scars with optical coherence tomography. Retina. 2009;29:631-7. 